\title{
Airway Management in Case of Recurrence of Pleomorphic Adenoma of Right Submandibular Gland and Soft Palate
}

\author{
Shivani Dalal*, Saibaba Thammishetty and Aruna Chandak \\ Department of Anaesthesiology, AVBRH, DMIMS, Sawangi, Wardha, Maharashtra, India; \\ shivani1363.sd@gmail.com, doctorchandak@gmail.com
}

\begin{abstract}
Objective: Pleomorphic adenoma is the most common type of all benign and malignant salivary gland tumours, involving more frequently the parotid gland. We present a case of a benign recurrent pleomorphic adenoma of the right submandibular gland and soft palate in a 42-year-old female without any signs of facial nerve involvement or damage. Methods: Case report. Findings: There was no evidence of the malignant transformation of the tumour confirmed on biopsy. It is $8 \mathrm{x} 4 \mathrm{~cm}$ oval in shape with diffuse border and was obstructing the upper airway and compression symptoms. The tumour was completely respected and reconstruction was done using pectoralis major myocutaneous (PMMC) flap. In this case, potential and predicted difficult airway is elaborated with excellent postoperative results. Applications/improvements: Though tumour of the submandibular gland is rare they require careful evaluation for the airway ventilation and intubation difficulties it poses.
\end{abstract}

Keywords: Pleomorphic Adenoma, Submandibular Gland, Soft Palate, Difficult Airway.

\section{Introduction}

Neoplasms which arise from salivary glands are rare and comprise only $1-4 \%$ of head face neck tumour, they represent wide variety of both benign and malignant histologic types. $10 \%$ of salivary gland tumour affect parotid gland,,$\underline{2}$ 5-10\% involve submandibular gland,, $1 \%$ involve sublingual gland, $\stackrel{1}{-} 5-15 \%$ involve minor glands. ${ }^{1}$ Pleomorphic adenoma accounts for $70-80 \%$ of benign salivary gland tumours and is common in the parotid gland. $\underline{3}$ Most common in the $3^{\text {rd }}-6^{\text {th }}$ decade of life. $\underline{3}$ Etiology is unclear ${ }^{2}$ with female sex predilection (F:M 2:1). $\underline{3}$ It is a solitary, mobile, slow-growing painless lesion. ${ }^{1}, \underline{3}$ Histologically myoepithelial cells are considered to be responsible for the production of extracellular matrix. ${ }^{4}$ Large tumours involving parapharyngeal space are predictors of the difficult airway. This case describes benign pleomorphic adenoma involving submandibular gland of right side with involvement of soft palate extending inti parapharyngeal space measuring $8 \times 4 \mathrm{~cm}$. A 42 year female patient of $70 \mathrm{~kg}$ weight, height- $163 \mathrm{~cm}$, presented to Sharad Pawar Dental College, Sawangi, Meghe, Wardha, with complain of painful swelling over lower right back region of jaw which was initially small and gradually increased in size of about $8 \times 4 \mathrm{~cm}$ approx. since 3 years associated localized dull aching intermittent pain. Association with -

- difficulty mastication,

- deglutition and

- difficulty in breathing in lying down position since 2 years, but symptoms became worse since 2 months she needed to be propped up position to maintain saturation percentage of oxygen (SPO2) while sleeping as it would cause airway obstruction,

${ }^{*}$ Author for correspondence 
- associated with hearing impairment over the right ear since $8-10$ days and

- change in voice since 1 month.

- was associated with excessive salivation

She was previously operated under general anaesthesia 3 years back (March 2016) but again after 5-6 months, she noticed swelling for which she did not opt for any treatment.

In 2018 (1 year back) she got her CT scan - mentioned below and FNAC right submandibular gland done s/o pleomorphic adenoma of the submandibular gland and soft palate.

No signs of facial nerve involvement noted, no palpable cervical/axillary/external iliac/inguinal lymph nodes noted.

Extraoral examination: large grossly asymmetrical due to swelling present over the lower right back region of jaw extending anterio-posteriorly from the right corner of the mouth to $1 \mathrm{~cm}$ posterior to angle of the angle of mandible and superior-lateral from ala-tragus line to $2 \mathrm{~cm}$ below the lower border of the mandible.

Size $-8 \times 4 \mathrm{~cm}$, Shape - roughly oval, Border - diffuse, Colour - same as adjacent area.

On palpation - tenderness absent, In duration present, Consistency - firm.

No evidence of bleeding or pus discharge present from it.

Intraoral examination: Adenoma was obstructing the upper airway by extending into parapharyngeal space and roof of nasopharynx displacing nasopharynx and oropharynx.

FNAC smear from swelling in the right submandibular area: stained with Pap stain show moderate cellularity with a few scattered or groups of epithelial cells, many groups/ sheets of myoepithelial cells and focal chondromyxoid matrix with blood in the background.

FNAC smear from swelling on the right side of the soft palate: stained with Pap stain show moderate cellularity with many groups/sheets of myoepithelial cells, a few scattered or groups of epithelial cells and focal chondromyxoid matrix with blood in the background.

No evidence of malignancy in the smear.

Preoperative investigation - CBC- $\mathrm{Hb}-10.2 \mathrm{gm} \%, \mathrm{WBC}$ - 9400/cu.mm, Platelet count -3.8 lacs/cu.mm.

Blood group and cross matching was done - A positive

Liver function test, Kidney function test and Thyroid function test were within normal limits

\section{Pulmonary Function Test Showed Normal Results}

\subsection{Mouth Opening Reduced $(25 \mathrm{~mm})$}

A single swelling present in lower right GB sulcus region extending from right retromolar region to alveolar ridge of left side involving soft palate bilateral vocal cord mobile, no pathology seen on vocal cords and pyriformis as shown in Figures 1 and 2. The large heterogeneously mass lesion in the region of the floor of the mouth on right appears to be arising from the right submandibular gland with extension in parapharyngeal spaces, roof of nasopharynx displacing and deviation of the wall of the nasopharynx and oropharynx. Postero-superiorly the mass is abutting deep lobe of the parotid gland and is extending into right masticator space. These as described above are s/o- pleomorphic adenoma. In an operated case

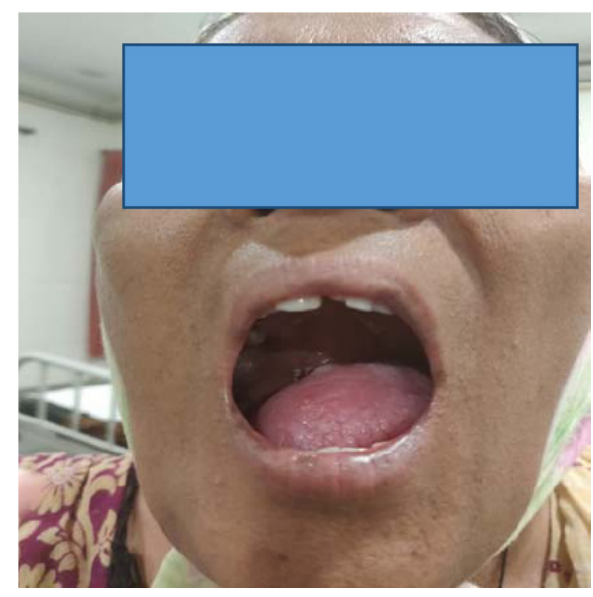

Figure 1. Preoperative - oral assessment.

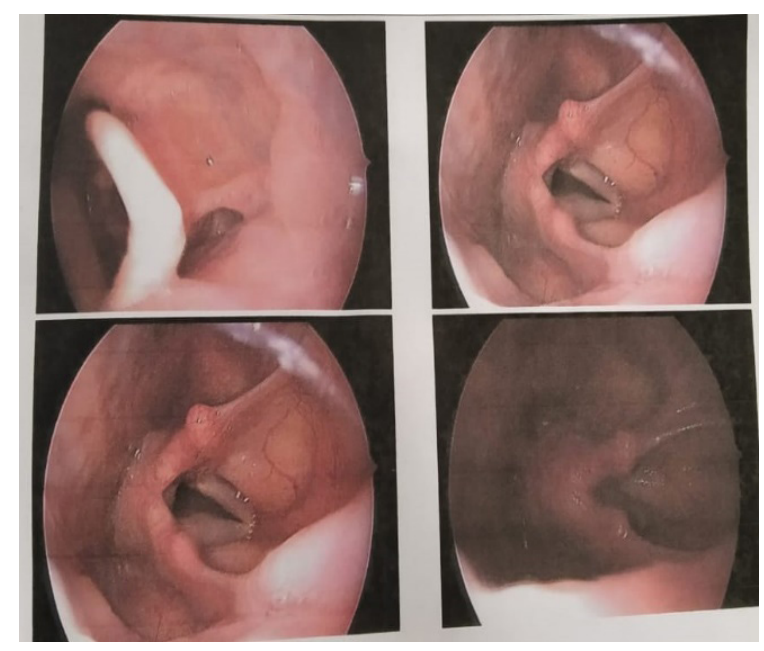

Figure 2. VDL. 
of submandibular gland mass - features suggestive of recurrence of mass as shown in Figures 3-5.
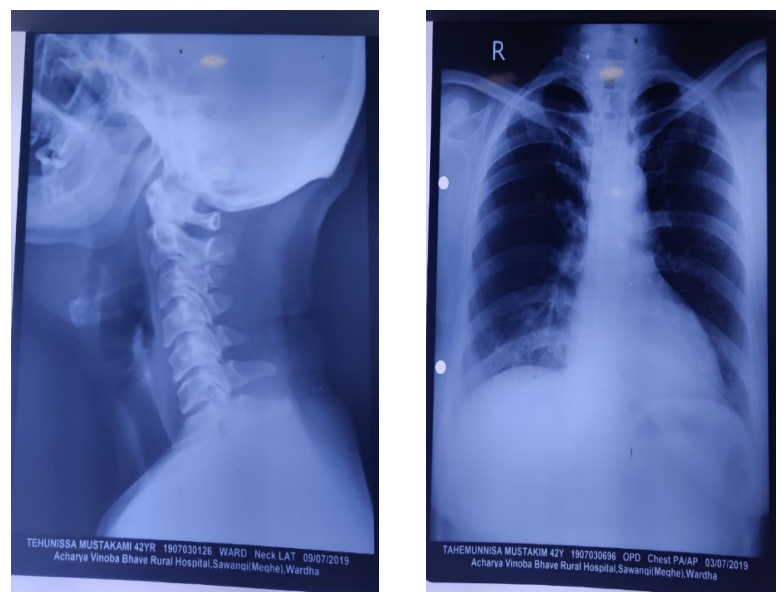

Figure 3. AP view neck-normal chest $\mathrm{x}$-ray $\mathrm{PA}$ view - normal.

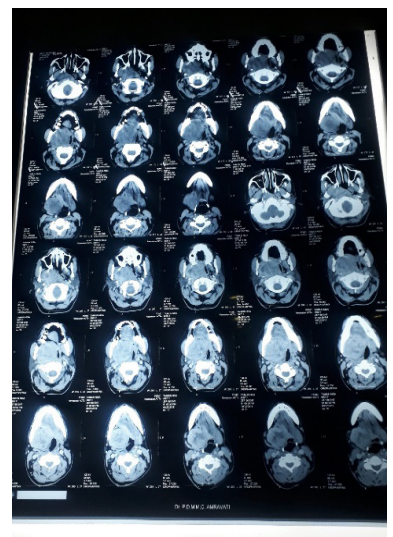

Figure 4. CT Scan PNS \& neck.

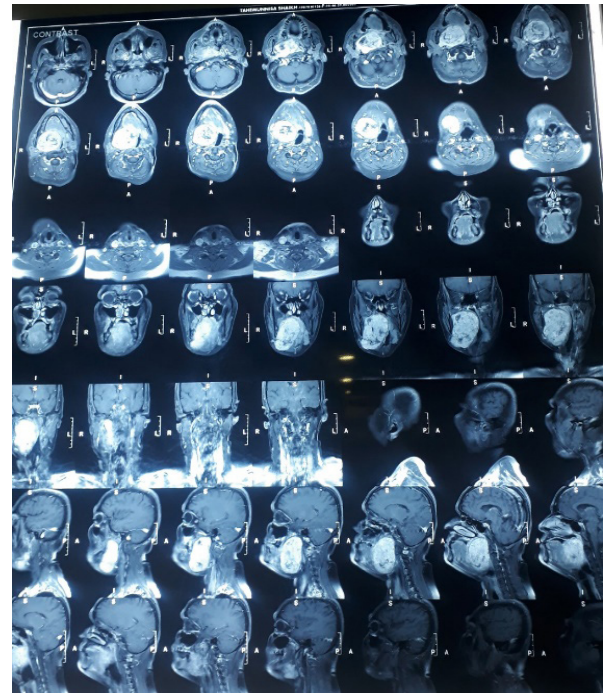

Figure 5. MRI right buccal mucosa.

\subsubsection{Preoperative $A B G$}

$\mathrm{pH}-7.40, \mathrm{pCO}_{2}-41.6$ mmhg, $\mathrm{pO}_{2}-91.9$ mmhg, $\mathrm{sO}_{2}-$ $97 \%$, p50 - 26.53, base $-1.3, \mathrm{Hco}_{3}-25.4$

\subsubsection{Preoperative Challenges}

- The patient had orthopnea - difficulty breathing in lying down position so needed head-up position every time she wanted to sleep as to maintain her SPO2 which use to fall as she tried sleeping without head up or straight, later she developed obstructive sleep apnea for which CPAP was given.

- as the tumour was extending into parapharyngeal space and roof of nasopharynx which was displacing nasopharynx and oropharynx causing upper airway obstruction making anticipated difficult intubation with McCoy blade or video laryngoscope blades. So the ultimate option was to go for Awake Fibreoptic Bronchoscopic intubation.

- The oral examination had reduced mouth opening with the absence of teeth in the lower jaw and protruded teeth in the upper jaw,

- Due to the presence of adenoma, it was difficult to assess mallampatti grading.

She was nebulized with salbutamol and budecortisone thrice daily till her day of surgery, chest physiotherapy was done, bedside PFT was within normal limits.

Consent for invasive airway management i.e cricothyroidotomy, tracheostomy and ventilator support was taken.

In the preoperative room, IV access was secured of $18 \mathrm{G}$ and IV fluid of ringer lactate solution was started, nebulization with Lox $4 \%$ was done, inj. Atropine $(0.01 \mathrm{mg} / \mathrm{kg})$ and Promethazine $(0.5 \mathrm{mg} / \mathrm{kg})$ was given intramuscularly. $\mathrm{O}_{2}$ mask was kept throughout.

Difficult airway trolley was prepared, emergency drugs like atropine, adrenaline, cardiovascular drugs, bronchodilators were kept. Anaesthesia was consent was taken during pre-anaesthetic evaluation. Awake intubation with fibreoptic was planned.

The patient was brought to operation theatre and multipara monitors were attached (Heart Rate, ECG, Blood Pressure (MAP), Respiratory Rate, ETCO2). The patient was maintaining in head-up position and a shoulder ramp was given before FOB. Airway blocks were given and awake fibre optic was performed, a $7 \mathrm{~mm}$ cuffed flexometallic endotracheal tube was inserted and 
tube placement was confirmed with FOB, ETCO2, chest auscultation and rise. Inj. Midazolam was given during the whole procedure until the placement of the tube. Once confirmed inj. Propofol $-2 \mathrm{mg} / \mathrm{kg}$ was given followed by muscle relaxation inj. Vecuronium $-0.1 \mathrm{mg} / \mathrm{kg}$, the patient was maintained on $\mathrm{O}_{2}$, sevoflurane, nitrous oxide.

After surgical excision of adenoma reconstruction with pectoralis major myocutaneus (PMMC) flap was done. Once the surgery was done patient was reversed and the probable plan was to leave the tube in situ but the patient could not tolerate the tube and was in severe distress, so extubation was done and no post-extubation respiratory compromise was noted as shown in Figures 6 and 7.

\section{Discussion}

Expected airway difficulty should be assessed preoperatively and planned accordingly and stepwise management should be planned if tracheal intubation fails. Difficult airway equipments including all sized ET tubes for appropriate age and smaller, FOB, cricothyroid puncture set, tracheostomy tubes and set, ventilating bougie was kept ready on the cart.

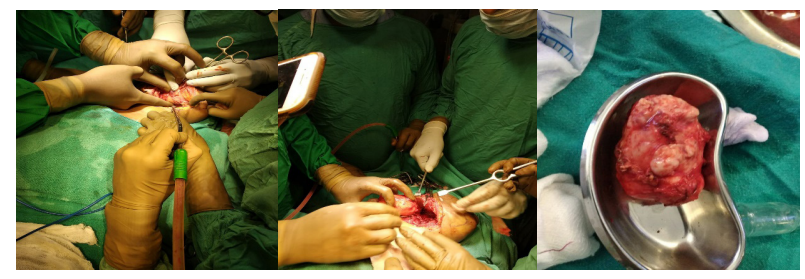

Figure 6. Pleomorphic adenoma was removed as depicted.

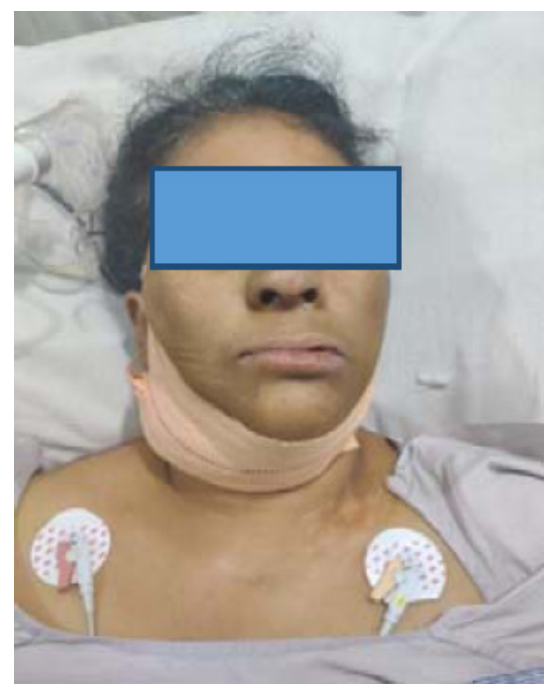

Figure 7. Post extubation in oral surgery ICU.
As awake intubation was planned, nasal preparation using otrivin drops and lignocaine jelly $2 \%$ was done. Proper airway blocks must be given to be able to tolerate FOB. For airway blocks - superior laryngeal nerve (SLN) block was given using $2 \%-2 \mathrm{ml}$ of lignocaine on either side of neck- greater cornua of hyoid bone is palpated (below angle of mandible) which is held between index and thumb finger and firm pressure is applied to displace it towards the side of block, a $25 \mathrm{G}$ needle is inserted to contact greater cornua of bone and then walked back and $2 \mathrm{ml}$ was injected on each side. Recurrent laryngeal nerve (RLN) block included inhalation with lox $4 \%-4 \mathrm{ml}$ and Tran tracheal block which was performed by insertion of a needle into the cricothyroid membrane until resistance lost (meant into larynx), aspiration was done, the air was aspirated $4 \%-4 \mathrm{ml}$ of lignocaine was injected.

Difficulties faced during intubation was-

- On the introduction of the fibreoptic bronchoscope, no airway anatomy was observed like hard palate/ soft palate/ posterior pillars/ subglottic area only a small slitlike opening was visible which was followed by

- difficulty in the negotiation of nasal flexometallic endotracheal tube from the nasopharynx to oropharynx as the adenoma was obstructing the pathway of ET tube, a prior nasopharyngeal airway was used to prevent airway trauma, the patient was intubated with a smaller size flexometallic endotracheal cuffed tube of no. $6.5 \mathrm{~mm}$.

- the patient was maintained on light sedation, no deep sedation was used due to anticipated difficulty intubation also high chances of upper airway obstruction by tongue fall and depression of respiration.

The only guidance of nasal ET tube was a small slitlike opening through which intubation was done, as anticipated difficult airway was present all the medication were given in the operation room itself premedication was also given in incremental dose first by giving intramuscular and then with inj. Glycopyrrolate - 0.004 $\mathrm{mg} / \mathrm{kg}$ due to its maximum anti-sialagogue activity, Inj. Midaz $-0.05 \mathrm{mg} / \mathrm{kg}$ was given prior to reducing anxiety.

Once nasal intubation was done with a $6.5 \mathrm{~mm}$ cuffed flexometallic endotracheal tube, bilateral chest rise was observed and air entry was checked along with the formation of ETCO2 which was confirmed by fibreoptic bronchoscopy. The patient was given inj. Fentanyl - $1 \mathrm{mcg} / \mathrm{kg}$, inducing agent - inj. Propofol $2 \mathrm{mg} / \mathrm{kg}$ and an intubating dose of muscle relaxant inj. 
Vecuronium- $0.1 \mathrm{mg} / \mathrm{kg}$ Patient was ventilated on volume control mode with TV - $8 \mathrm{ml} / \mathrm{kg}, \mathrm{RR}-16 / \mathrm{min}$, PEEP $5 \mathrm{~cm} \mathrm{H}_{2} \mathrm{O}$, I:E-1:2, maintained on $\mathrm{O}_{2}, \mathrm{~N}_{2} \mathrm{O}$, sevoflurane and vecuronium top-ups.

The patient's vitals were unremarkable, analgesia was given with inj. Fentanyl $-1 \mathrm{mcg} / \mathrm{kg}$ and later at the near end of surgery inj. Paracetamol - 20mg/kg. No complications were noted intraoperatively

Once the adenoma was removed and closure was done, the probable plan was to keep patient nasally intubated keeping in mind about obstruction of the airway by pharyngeal pouch as the majority of tumours was obstructing the airway which had lead to difficulty in fibreoptic intubation.

But the patient could not tolerate tube and so extubation was done under strict guidance where difficult airway trolley was arranged along with an emergency tracheostomy set. No post-extubation respiratory compromise was noted.

\section{Conclusion}

Though tumour of the submandibular gland is rare they require careful evaluation not only for surgical excision but also for the airway ventilation and intubation difficulties it poses.
Care needs to be taken prior to shifting the patient to the operating room.

Preoperatively detailed evaluation of extending of tumour, any compression symptoms and airway difficulty needs to be ruled out.

Intraoperatively nasal ETtube placement and its displacement along with maintenance of vitals are to be done.

Postoperatively care needs to be taken to prevent respiratory complications posing reflection of the difficult airway.

\section{References}

1. Patil P, Burde K, Naikmasur VG, Thorawat A. Pleomorphic adenoma of submandibular gland: A case report with review of literature. Dent Res J. 2014;11(3):411-4.

2. Bagga $M$, Bhatnagar $D$, Bhatnagar D. An unusual presentation of pleomorphic adenoma: a case report. J Indian Acad Oral Med Radiol. 2016;28(2);191-4.

3. Zhan KY, Khaja SF, Flack AB, Day TA. Benign parotid tumors. Otolaryngol Clin North Am. 2016;49(2):327-42.

4. Auclair PL. Salivary gland neoplasms: general considerations. In: Surgical pathology of the salivary glands; 1991. P. 135-64. 